\title{
Trails of Mobile Majoranas in an Iron Chalcogenide?
}

\section{Signature of Dispersing 1D Majorana Channels in an Iron-based Superconductor}

Authors: Zhenyu Wang, Jorge Olivares Rodriguez, Martin Graham, G. D. Gu, Taylor Hughes, Dirk K. Morr, and Vidya Madhavan

arXiv:1903.00515

\section{Evidence for Helical Hinge Zero Modes in an Fe-Based Superconductor} Authors: Mason J. Gray, Josef Freudenstein, Shu Yang F. Zhao, Ryan O'Connor, Samuel Jenkins, Narendra Kumar, Marcel Hoek, Abigail Kopec, Takashi Taniguchi, Kenji Watanabe, Ruidan Zhong, G. D. Gu, and K. S. Burch arXiv:1902.10723

\section{Recommended with a Commentary by Fa Wang, Peking University, Beijing, China}

The iron chalcogenide $\mathrm{FeTe}_{1-x} \mathrm{Se}_{x}$ has become a promising platform for the realization of localized Majorana zero modes (see previous commentaries [1,2]). This material hosts bulk nodeless superconductivity [3], and surface states similar to those of 3D topological insulators $[4,5]$. These traits actually imbue this material with even greater potentials. The two recommended papers report experimental evidence that $\mathrm{FeTe}_{1-x} \mathrm{Se}_{x}$ might also host dispersing 1D helical Majorana fermions on certain domain walls [6] or hinge structures [7].

In $2008 \mathrm{Fu}$ and Kane [8] proposed that 1D helical Majorana fermions can exist in a " $\pi$ phase shift domain wall" of superconductors, with a phase jump of $\pi$ of the pairing order parameter, in proximity to the surface states of a 3D topological insulator (see Fig. 1). The topological surface states with nodeless superconducting gap in $\mathrm{FeTe}_{1-x} \mathrm{Se}_{x}$ [4] set up a natural experimental stage for this proposal. In the first recommended preprint [6], the authors observed structural domain walls with "half-unit cell shift" by Fourier analysis of the STM topography image (see Fig. 2). The two domains are related by a "half-unit cell shift", or the "glide plane symmetry", in $\mathrm{FeTe}_{1-x} \mathrm{Se}_{x}$. The glide plane symmetry in ironbased superconductors is the translation by the displacement of two nearest-neighbor Fe atoms, followed by a mirror reflection about the Fe plane ( $a b$-plane). This symmetry allows us to use the "unfolded" 1-Fe-per-cell Brillouin zone (strictly speaking only for $k_{z}=0$ or $\pi$, because $k_{z}$ changes sign under this symmetry). 

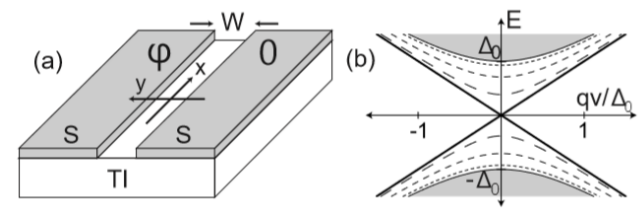

Figure 1: Left: A " $\pi$-phase shift domain wall" (when the phase difference $\phi$ equals to $\pi$ ) of superconductors $(\mathrm{S})$ in proximity to a topological insulator(TI). Right: The dispersion of the bound states on the domain wall. Solid lines with helical dispersion are for $\phi=\pi$. Taken from Ref. [8].
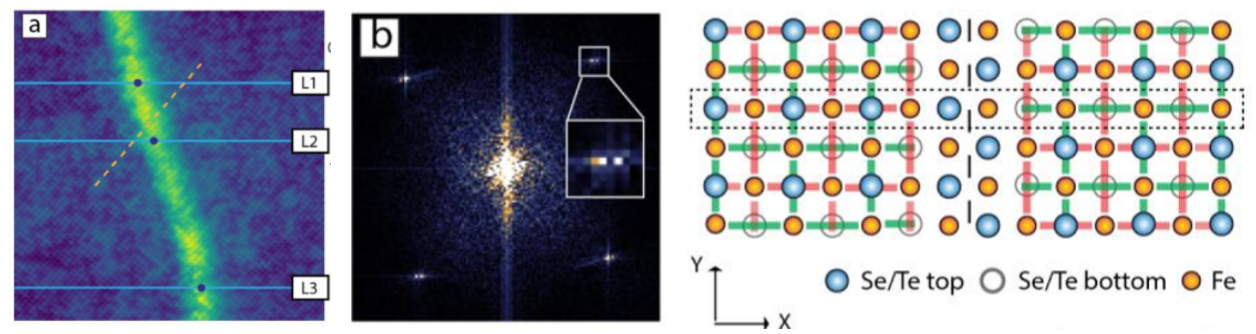

Figure 2: Left: The topography image for a region covering a domain wall. Center: The Fourier transform of the left image. The split Bragg peaks are produced by interference of the Fourier components of the two domains. Right: Schematic picture of the "half-unit cell shift" domain wall (dashed line). Although the Fe atoms still form a square lattice, glide plane symmetries are broken by the domain wall. Taken from Ref. [6].

The authors of [6] then performed low temperature $\left(0.3 \mathrm{~K}\right.$, far below the $T_{c}$ of $\left.14.5 \mathrm{~K}\right)$ scanning tunneling spectroscopy(STS) around these "half-unit cell shift" domain walls. The $\mathrm{dI} / \mathrm{dV}$ spectrums on these domain walls show a "flat density of states" (see Fig. 3), while the spectrums far away from the domain walls have a full superconducting gap consistent with previous studies $[3,5]$. By spatial distributions of the $\mathrm{dI} / \mathrm{dV}$ spectrums, the authors concluded that these low energy density of states are from bound states at the domain wall, with a localization length of 3nm at Fermi level. These "flat density of states" are not observed on other types of 1D defects, including step edges in this material and twin-domain walls in FeSe. They then made a bold proposal that the "half-unit cell shift" domain walls realize the " $\pi$-phase shift domain wall" in the theoretical setup of Fu and Kane [8] and the observed "flat density of states" are from the helical Majorana fermions on these domain walls.

Although the story is plausible, several pertinent questions should be asked:

- How can this structural domain wall induce a $\pi$-phase shift of the superconducting order parameter? The authors invoked a theory of "odd-parity spin-singlet pairing" in ironbased superconductors, in which the pairing order parameter has momentum $(\pi, \pi)$ in the unfolded Brillouin zone, and therefore will change sign under the glide plane symmetry relating the two structural domains. However, this symmetry consideration alone cannot determine the relative phase of the two superconducting regions; energetic considerations would be more convincing to show, for example by self-consistent BdG 

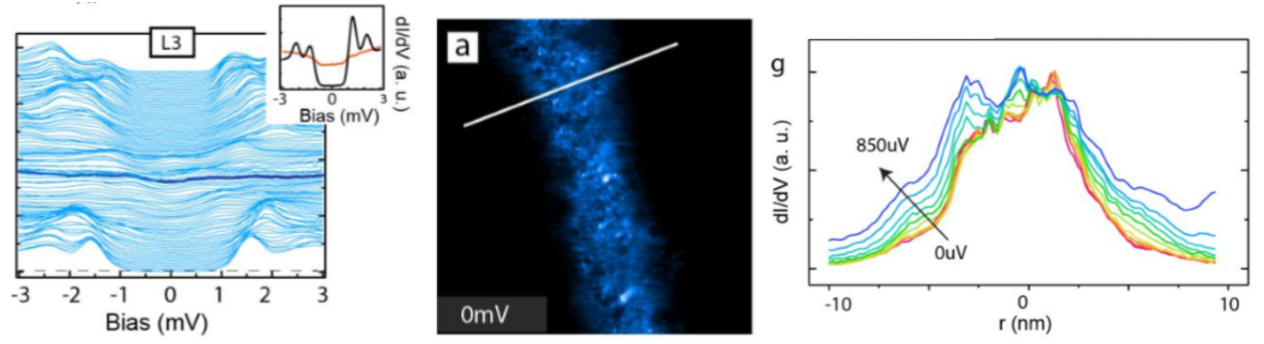

Figure 3: STS dI/dV spectrums taken along a cut (see Fig. 2) across the domain wall. The highlighted spectrum is taken on the domain wall, showing a flat density of states. Inset compares the spectrums on and far away from the domain wall. Center and right: Spatial distributions of the $\mathrm{dI} / \mathrm{dV}$ spectrum showing localized density of states around the domain wall. Taken from Ref. [6].

solutions, that the superconducting order parameter indeed changes sign across this domain wall.

- How can the assumed helical Majorana fermions produce a flat density of states(DOS) that seems to recover the normal state DOS (including the topological trivial bulk states)? In the numerical simulations by the authors, using $\mathrm{s}_{p m}$ paring instead of the "odd-parity spin-singlet pairing", with a $\pi$-phase shift across the domain wall enforced by hand, the density of states at the domain wall is not a simple flat DOS. It would be more convincing if the authors can measure the temperature evolution (across Tc) of the flat DOS to distinguish it from the normal state DOS of a non-superconducting region.

We now turn to the second recommended preprint [7]. The authors have made normal metal/superconductor junctions on the hinges between the (001) and (100) surfaces of exfoliated flakes of $\mathrm{FeTe}_{1-x} \mathrm{Se}_{x}$ single crystals, and observed strong zero-bias conductance peaks(ZBCP) below Tc, while junctions not in contact with the hinges do not show strong ZBCP (see Fig. 4).
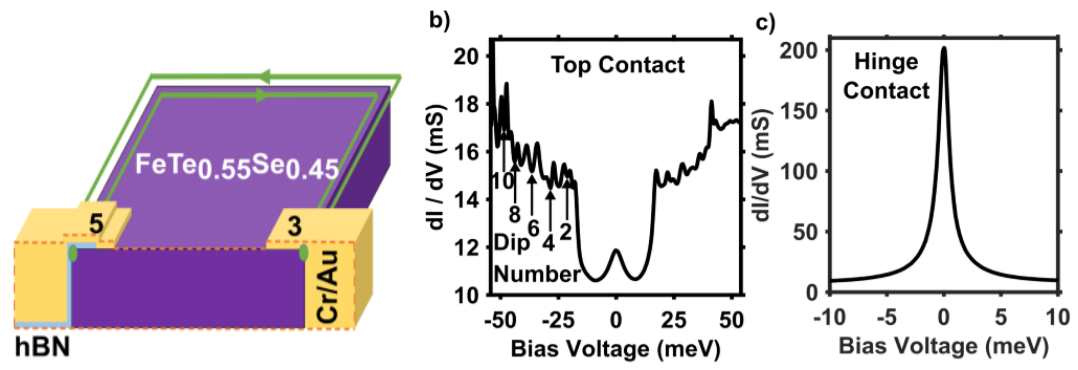

Figure 4: Left: Schematic picture of normal metal/superconductor junctions in contact with the hinge (labeled by "3") and not in contact with the hinge (labeled by "5"). Center: Conductance for the junction not in contact with the hinge. Right: Conductance for the junction in contact with the hinge, showing strong ZBCP. Taken from [7]. 
It is claimed that this ZBCP is the evidence of a recent theoretical proposal of "helical hinge Majorana fermions" [9]. This theory [9] proposed that superconductors with unconventional $\mathrm{s}_{p m}$ pairing and topological surface states are "higher-order topological superconductors", with symmetry-protected helical Majorana fermions on the hinges between (001) and (100) surfaces. The basic idea is that the bulk $\mathrm{s}_{p m}$ pairing may induce pairing order parameters with opposite signs on the two surfaces, and the hinge line between the two surfaces become a " $\pi$-phase shift domain wall" (see Fig. 5 ).
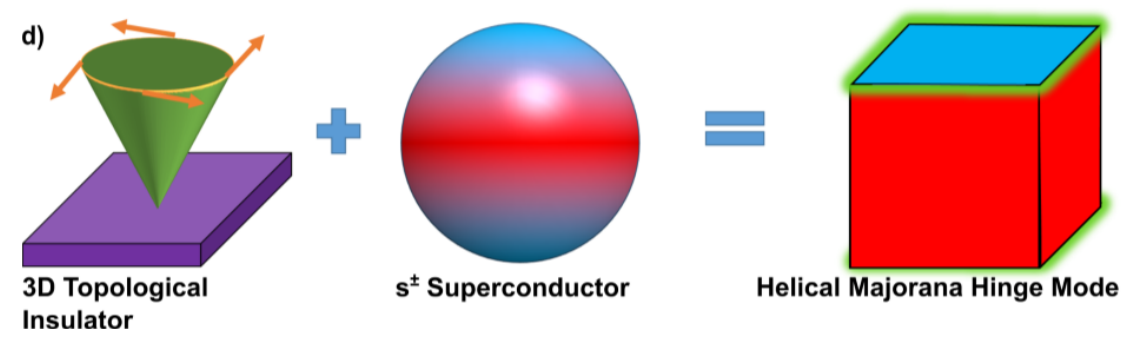

Figure 5: Schematic picture for the idea that topological surface states plus $\mathrm{s}_{p m}$ pairing can have "helical Majorana hinge modes". Red and blue colors denote opposite signs of the paring order parameter. Taken from [7].

The theoretical idea is indeed reasonable. However, the authors do not explain how the simply assumed helical Majorana fermions, with a roughly constant density of states (see e.g. [6]), can produce a ZBCP. In the case of candidate topological superconductor $\mathrm{Cu}_{x} \mathrm{Bi}_{2} \mathrm{Se}_{3}$, rather complex dispersions of the surface Majorana fermions have to be assumed to explain the observed ZBCP [10].

Are the results of Ref. [6, 7] really trails of mobile Majorana fermions in $\mathrm{FeTe}_{1-x} \mathrm{Se}_{x}$ ? I think this is certainly an interesting question that deserves further study and careful scrutiny.

\section{References}

[1] Fa Wang, "Iron-based superconductors went topological ?", Journal Club for Condensed Matter Physics, January, 2018. Available at https://www.condmatjclub.org/?p=3286.

[2] Patrick A. Lee, "Spontaneous vortex formation and Majorana zero mode in iron based superconductor", Journal Club for Condensed Matter Physics, December, 2018. Available at https://www.condmatjclub.org/?p=3496.

[3] T. Hanaguri, et al, Science 328, 474 (2010).

[4] Peng Zhang, et al, Science 360, 182 (2018).

[5] Dongfei Wang, et al, Science 362, 333 (2018).

[6] Zhenyu Wang, et al, arXiv:1903.00515.

[7] Mason J. Gray, et al, arXiv:1902.10723. 
[8] Liang Fu, and C. L. Kane, Phys. Rev. Lett. 100, 096407 (2008).

[9] Ruixing Zhang, et al, Phys. Rev. Lett. 122, 187001 (2019).

[10] Timothy. H. Hsieh, and Liang Fu, Phys. Rev. Lett. 108, 107005 (2012). 